\title{
MyelTracer: A Semi-Automated Software for Myelin g-Ratio Quantification
}

\author{
Tobias Kaiser, ${ }^{1,2, *}$ Harrison Mitchell Allen, ${ }^{1,3, *}$ Ohyoon Kwon, ${ }^{1,2, *}$ Boaz Barak, ${ }^{4}$ Jing Wang, ${ }^{5}$ Zhigang He, $^{5}$ \\ Minqing Jiang, ${ }^{1,2}$ and ${ }^{-G u o p i n g ~ F e n g ~}{ }^{1,2,6}$
}

https://doi.org/10.1523/ENEURO.0558-20.2021

\begin{abstract}
${ }^{1}$ McGovern Institute for Brain Research, Cambridge, MA 02139, 2Department of Brain and Cognitive Sciences, Massachusetts Institute of Technology, Cambridge, MA 02139, ${ }^{3}$ Department of Electrical Engineering and Computer Science, Massachusetts Institute of Technology, Cambridge, MA 02139, ${ }^{4}$ The Sagol School of Neuroscience and The School of Psychological Sciences, Tel Aviv University, Tel Aviv, 6997801, Israel, ${ }^{5}$ F.M. Kirby Neurobiology Center, Boston Children's Hospital, and Department of Neurology and Ophthalmology, Harvard Medical School, Boston, MA 02115, and ${ }^{6}$ Stanley Center for Psychiatric Research, Broad Institute of MIT and Harvard, Cambridge, MA 02142
\end{abstract}

\section{Visual Abstract}
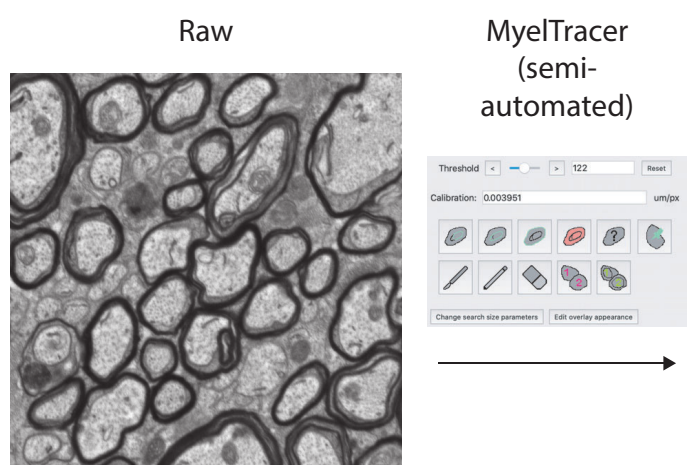

MyelTracer overlay output

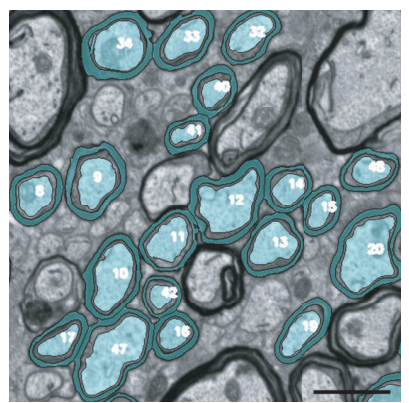

Output matches manual measurement

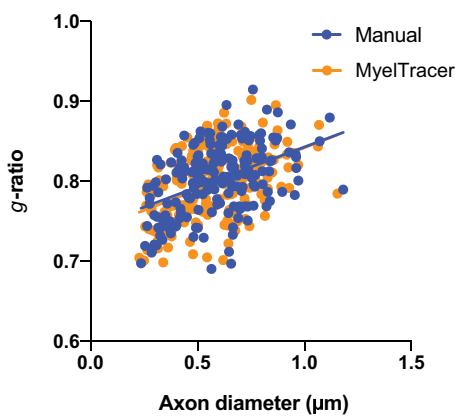

In the central and peripheral nervous systems, the myelin sheath promotes neuronal signal transduction. The thickness of the myelin sheath changes during development and in disease conditions like multiple sclerosis. Such changes are routinely detected using electron microscopy through $g$-ratio quantification. While $g$-ratio is one of the most critical measurements in myelin studies, a major drawback is that $g$-ratio quantification is extremely laborious and time-consuming. Here, we report the development and validation of MyelTracer, an installable, stand-alone software for semiautomated g-ratio quantification based on the Open Computer Vision Library (OpenCV). Compared with manual g-ratio quantification, using MyelTracer produces consistent results across multiple tissues and animal ages, as well as in remyelination after optic nerve crush, and reduces total quantification time by $40-60 \%$. With $g$-ratio measurements via MyelTracer, a known hypomyelination phenotype can be detected in a Williams syndrome mouse model. MyelTracer is easy to use and freely available for Windows and Mac OS X (https://github.com/HarrisonAllen/MyelTracer).

Key words: electron microscopy; g-ratio; myelin; toolbox

\section{Significance Statement}

An easy-to-use software suite for $g$-ratio quantification in myelin ultrastructure micrographs is currently unavailable, but it is much needed to streamline the study of myelin in homeostasis and disease-related conditions. We used computer vision libraries to develop a freely available software to facilitate studies of myelination. 


\section{Introduction}

The myelin sheath is essential for proper neuronal functions in central and peripheral nervous systems. Oligodendrocytes and Schwann cells produce the myelin sheath through lamellar enwrapping of axons (Simons and Trotter, 2007; Salzer, 2015; Hughes and Appel, 2016; Stadelmann et al., 2019). The myelin sheath insulates axons, thereby preserving axonal integrity and promoting neuronal signal transduction (Sherman and Brophy, 2005; Nave, 2010). Under physiological conditions, changes in myelin contribute to behaviorally relevant neural plasticity mechanisms and experience-dependent sensory adaptations (Gibson et al., 2014; McKenzie et al., 2014; Hughes et al., 2018).

Abnormal changes in myelin are prominent features of many clinical pathologies. Ultrastructural myelin abnormalities, such as hypomyelination, myelin degeneration, and tomaculi, are cardinal features of prototypic white-matter diseases like multiple sclerosis, Pelizaeus Merzbacher disease, and Charcot-Marie-Tooth disease (Krajewski et al., 2000; Sander et al., 2000; Franklin and ffrench-Constant, 2008; Lin and Popko, 2009; Duncan and Radcliff, 2016). In addition, myelin abnormalities have recently been discovered as common features of neurodevelopmental disorders, including Pitt-Hopkins syndrome, Rett syndrome, autism spectrum disorders (ASDs), and William syndrome (Zhao et al., 2018; Barak et al., 2019; Phan et al., 2020).

In both preclinical and clinical settings, pathologic myelin abnormalities are often identified by studying myelin ultrastructure. Specifically, researchers use $g$-ratio as a metric for the relative thickness of the myelin sheath in cross-sectional micrographs (Rushton, 1951). Since manual myelin tracing is a major bottleneck in $g$-ratio quantification, tools that streamline this process will tremendously facilitate future studies investigating activity-dependent homeostatic and pathologic changes to the myelin sheath.

Received December 23, 2020; accepted June 17, 2021; First published June 24, 2021.

The authors declare no competing financial interests.

Author contributions: T.K., H.M.A., M.J., and G.F. designed the research. H. M.A. wrote the software; T.K. and M.J. generated the electron microscopy data; T.K., M.J., and O.K. analyzed the data and benchmarked the software. B. B., J.W., and Z.H. contributed electron micrographs for additional analysis. T. K., M.J., and G.F. wrote the paper with inputs from all authors.

T.K. is supported by the Tan-Yang center for Autism Research at MIT. G.F. is supported by the National Institute of Mental Health Grant 5R01MH097104, the Poitras Center for Affective Disorders Research at MIT, Tan-Yang Center for Autism Research at MIT, Stanley Center for Psychiatric Research at Broad Institute of MIT and Harvard, Nancy Lurie Marks Family Foundation, Simons Foundation Autism Research Initiative (SFARI), and Simons Center for the Social Brain at MIT.

${ }^{*}$ T.K., H.A., and O.K. contributed equally to this work.

Acknowledgements: We thank all members of the Feng lab for helpful discussions. We also thank Nicholas Sanders and Dongqing Wang for excellent technical support, and Maria Ericsson and Louise Trakimas at the Harvard Electron Microscopy Core facility.

Correspondence should be addressed to Minqing Jiang at minqing@mit.edu or Guoping Feng at fengg@mit.edu.

https://doi.org/10.1523/ENEURO.0558-20.2021 Copyright @ 2021 Kaiser et al.

This is an open-access article distributed under the terms of the Creative Commons Attribution 4.0 International license, which permits unrestricted use, distribution and reproduction in any medium provided that the original work is properly attributed.
Several non-automated, semi-automated, and fully automated toolboxes are currently available. They include G-ratio for ImageJ, AxonSeg, and AxonDeepSeg (Goebbels et al., 2010; More et al., 2011; Bégin et al., 2014; Zaimi et al., 2016, 2018; Janjic et al., 2019). Collectively, these tools have facilitated different quantitative measurements of myelin ultrastructure, such as the number of axons and g-ratio. However, the current tools with more automation impose difficult requirements on their users, such as preprocessing data or modifying parameters by writing code. These drawbacks have impeded the tools' widespread adoption by the scientific community. We identified four major requirements of an open access toolbox that would significantly facilitate myelin analyses: (1) an intuitive graphical user interface that can be installed and used without running code; (2) consistent quantification results compared with manual analysis; (3) significantly less time-consuming; and (4) well-organized output data files and overlay images for publication and post hoc quality control.

Here, we report the development and validation of MyelTracer, an easy-to-use software that fulfills these critical requirements. Built using OpenCV and PyQt5's GUI toolkit, MyelTracer replaces the manual tracing of axons and myelin with semi-automated, threshold-dependent outlining. Compared with manual quantification, semi-automated quantification of axon diameters and $g$-ratio via MyelTracer produces consistent results across several tissues and developmental time points while reducing total quantification time by $40-60 \%$ depending on the region. Furthermore, using $g$-ratio measured with MyelTracer, we can detect a known hypomyelination phenotype in a Williams syndrome mouse model, as well as in remyelination after optic nerve crush, indicating the applicability of MyelTracer for studies in disease contexts. To ensure MyelTracer's all-in-one output functionality, we included a manual tracing function and counters for quantification of abnormally shaped sheaths and fraction of myelinated axons, respectively. MyelTracer is a valuable tool for the study of myelin ultrastructure and is freely available to the public as an installable software for Mac and PC (https:// github.com/HarrisonAllen/MyelTracer).

\section{Materials and Methods}

\section{Animal work}

All animal procedures were performed in accordance with the Massachusetts Institute of Technology animal care committee's regulations.

\section{Code accessibility}

The code/software described in the paper is freely available online at https://github.com/HarrisonAllen/ MyelTracer. The code is available as Extended Data 1.

\section{Software development}

Outline extraction of axons is performed using an implementation of OpenCV in Python. The processing is done as follows. First, the color space of the image is converted 
from RGB to grayscale, so that all pixels have a scalar magnitude between 0 and 255 .

cvtColor(src, COLOR_BGR2GRAY)

Then, a bilateral filter is applied to the source image to reduce noise and smooth edges.

bilateralFilter(src, $d=9$, sigmaColor $=75$, sigmaSpace $=75$ )

A binary thresholding filter converts pixel values below the user defined threshold, $t$, to black, and those above $t$ to white.

threshold(src, thresh=t, maxval $=255$, type $=$ THRESH BINARY)

Lines drawn by the user are then applied to the thresholded image using the polylines function.

Contours are extracted as the boundaries between the black and white pixels of the thresholded image.

findContours(src, mode=RETR_TREE, method=CHAIN APPROX SIMPLE)

Contours with fewer than five vertices are filtered out using the len function as well as contours that do not fall within the user-defined area constraints, Min Size and Max Size, calculated using the contourArea function.

Once selected, contours for each feature, axon, inner myelin sheath, and outer myelin sheath, are grouped together by checking whether the features nest within one another using pointPolygonTest.

Exported data are written in a plain-text csv document for compatibility with most spreadsheet softwares. The exported features are organized by the order in which the user selected the axon feature. Area is calculated using contourArea, perimeter is calculated using arcLength, diameter is calculated using $2 \cdot \sqrt{\text { area } / \pi}$, and g-ratio is calculated using $\sqrt{\text { innerMyelinArea/OuterMyelinArea. }}$

The extraction software is wrapped in a custom-designed PyQt5 GUI, and both Windows and MacOS installers are generated using the fman Build System (fbs). MyelTracer has been tested and proven to work on Windows 10 and MacOS Catalina 10.15.

\section{Electron microscopy and $\boldsymbol{g}$-ratio tracing \\ Sample preparation and imaging}

One- and two-month-old male and female C57BL/6J mice were deeply anesthetized with isoflurane and transcardially perfused with $30 \mathrm{ml}$ ice-cold saline solution followed by $30-\mathrm{ml}$ fresh ice-cold $2.5 \%$ glutaraldehyde $+2 \%$ PFA in $0.1 \mathrm{~m}$ sodium cacodylate buffer $(\mathrm{pH} 7.4)$. Brains, optic nerves and sciatic nerves were dissected and kept in the fixation solution overnight at $4^{\circ} \mathrm{C}$. Small pieces (1to 2-mm cubes) of tissue samples from the dorsal corpus callosum at the level of the fornix (bregma $-0.94 \mathrm{~mm}$ ), optic nerve, and sciatic nerve were postfixed for at least $2 \mathrm{~h}$ at room temperature in the fixation solution. They were then washed in $0.1 \mathrm{~m}$ sodium cacodylate buffer and postfixed with $1 \%$ osmium tetroxide/ $1.5 \%$ potassium ferrocyanide for $1 \mathrm{~h}$ before being washed in water three times and incubated in $1 \%$ aqueous uranyl acetate for $1 \mathrm{~h}$. This was followed by two washes in water and subsequent dehydration in grades of alcohol (10 min each; 50, 70, 90 and $2 \times 10 \mathrm{~min} 100 \%)$. The samples were then put in propylene oxide for $1 \mathrm{~h}$ and infiltrated overnight in a 1:1 mixture of propylene oxide and epoxy resin mixture (TAAB Epon; Marivac Canada). The following day, the samples were embedded in TAAB Epon and polymerized at $60^{\circ} \mathrm{C}$ for $48 \mathrm{~h}$. Ultrathin sections $(\sim 60 \mathrm{~nm})$ were cut on a Reichert Ultracut S microtome (Leica), picked up on copper grids stained with lead citrate and examined in a JEOL 1200EX transmission electron microscope (JEOL USA). The images were recorded with a $2 \mathrm{k}$ CCD camera (Advanced Microscopy Techniques).

\section{Quantification of $g$-ratio}

To compare manually quantified $g$-ratio and those quantified with MyelTracer, three images for each region of interest were analyzed manually and using MyelTracer. Each image was analyzed first with MyelTracer, which automatically numbered every quantified axon. For manual $g$-ratio quantification, the same axons were manually numbered and then traced using ImageJ. To compare the control and Gtf2i-knock-out group, raw images were obtained from Barak and coworkers (Barak et al., 2019), blinded of their genotypes and quantified using MyelTracer. To test MyelTracer's ability to quantify $g$-ratios in remyelinating tissues, raw images of the optic nerve from control animals and animals $28 \mathrm{~d}$ post-injury with Montelukast and Pexidartinib treatment were obtained from Wang et al. (2020). For each experiment comparing manual tracing with MyelTracer, several micrographs were obtained from preparations of two to three mice per anatomic region. Within these micrographs, at least 100-300 axons were analyzed depending on the anatomic region. For testing MyelTracer's capability to detect a hypomyelination phenotype in a Williams syndrome model, several micrographs from three mice per genotype with a total of 332 and 455 axons were analyzed for control and Gtf2i fl/fl; Nex-Cre mice, respectively. For optic nerve, sciatic nerve, and corpus callosum, three different researchers recorded image quantification times. Numbers denoting measured axons in representative images were enlarged for easier visualization. MyelTracer was run on a MacBook Pro $(2.8 \mathrm{GHz}, 16 \mathrm{~GB}$ RAM, Intel Iris Plus Graphics $6551536 \mathrm{MB}$ and macOS Catalina version 10.15.2.), Windows 10 (Intel Core i5-7300HQ CPU $2.50 \mathrm{GHz}$, 8GB RAM, NVIDIA GeForce GTX 1050 Ti), and MacBook Air (Intel Core i5 1.3 GHz CPU, 4GB RAM, Intel HD Graphics 5000, macOS Catalina version 10.15.2).

\section{Results}

\section{MyelTracer is a semi-automated software using computer vision}

G-ratio quantification requires measuring the crosssectional areas of axons and myelin sheaths, which is typically performed via manual tracing. Done manually, this process can be immensely time-consuming and laborious. To facilitate g-ratio quantification, we created MyelTracer by wrapping the powerful Python Iibraries provided by OpenCV in an easy-to-use GUI using PyQt5. MyelTracer performs automatic detection of contours using dynamic thresholding and separation of adjacent myelin sheaths based on user input. We packaged MyelTracer as a readily installable 
software for Windows and Mac OS X operating systems. Conceptually, MyelTracer takes a raw electron micrograph input image (Fig. 1A), converts it to grayscale, applies a bilateral filter, and thresholds it to a black and white binary image (Fig. 1B). MyelTracer extracts boundaries between the black and white pixels on the binary image and creates a contour image based on user-defined filtering parameters (Fig. 1C). For $g$-ratio plotting, MyelTracer is configured to measure the inner and outer myelin sheath as well as the axon diameter to account for periaxonal space present in electron micrographs (Fig. 1A). Using the GUI (Fig. $1 G)$, the user can select the contours of axon, inner myelin, and outer myelin while adjusting the threshold to closely match the displayed contours to the underlying myelin ultrastructure. Once selected, MyelTracer groups the corresponding axons, inner myelin, and outer myelin together and overlays those selections on top of the original image (Fig. 1D). The software automatically computes diameters of perfect circles from area measurements and derives $g$-ratios by dividing inner myelin diameters by outer myelin diameters, which can then be plotted against the axon diameter (Fig. 1E,F).

To use MyelTracer efficiently, we recommend the following workflow (Fig. 1H, Extended Data 2). First, select all axons in the image by adjusting the threshold parameter to match contours to the axon shape. Second, adjust the threshold to match myelin sheaths. Third, check for contiguous contours of adjacent myelin sheaths as well as discontinuous contours around myelin sheaths. If necessary, use the cut tool to separate adjacent myelin sheaths, or use the draw tool to connect discontinuous myelin sheaths. Fourth, select the inner and outer myelin sheath contours, adjusting the threshold parameter to fit. Fifth, save the progress and export the result, which includes $g$-ratio measurements and an overlay (Fig. 1D). MyelTracer also provides a manual tracing option for otherwise untraceable features and a counting tool to calculate the percentage of myelinated axons. Together, these functions make MyelTracer an all-in-one software suite for studying myelin ultrastructure.

\section{MyelTracer saves quantification time while maintaining accuracy}

Myelin $g$-ratio quantification is routinely done by manually tracing the myelin sheath. Manual tracing is very time consuming but considered the standard practice for accurate results. To compare the accuracy of MyelTracer to manual tracing in moderately myelinated tissues, we measured $g$-ratios using both methods in the same electron micrographs of corpus callosum from one-month-old mice (Fig. 1/). Plotting the $g$-ratio as a function of axonal diameter, we did not find a significant difference between MyelTracer and manual quantification results (Fig. 1J, MyelTracer: slope 0.061, y-intercept 0.77, $n=173$, manual: slope $0.057, y$-intercept $0.77, n=173$; slopes $p=$ 0.8996 , intercept $p=0.7044^{\mathrm{a}}$ ) and their averages (Fig. $1 \mathrm{~K}$, MyelTracer mean \pm SD: $0.8072 \pm 0.04417, n=173$, manual: $\left.0.8066 \pm 0.04220, p=0.9045^{\mathrm{b}}\right)$. Next, to examine the amount of quantification time MyelTracer saves, the average amount of quantification time spent per axon was compared between these two methods. Using MyelTracer, significantly less time was spent per axon compared with manual tracing (Fig. 1L, MyelTracer mean: 0.593, manual: mean 1.0, $n=173$ axons, three images, $p=0.0069^{\circ}$ ). Further, to exemplify that MyelTracer can trace myelin sheets containing folds, several such sheets were analyzed. Taking advantage of the cut tool, MyelTracer readily detects myelin folds and correctly quantifies the myelin sheet without including the area in the fold (Fig. 1M-P). Together, these data show that MyelTracer maintains a high level of accuracy, comparable to manual tracing, while saving $\sim 40 \%$ of time for the user.

Myelination is spatiotemporally regulated (Mayoral and Chan, 2016; Sun et al., 2018). As a result, there are variations in myelin sheath thickness and spatial density of myelinated axons across different types of tissues and developmental time points. To assess the feasibility of using MyelTracer for different types of tissue samples, electron micrographs from optic nerves and sciatic nerves were examined (Fig. 2A,E). In the optic nerve of twomonth-old mice, plotting $g$-ratio as a function of axon diameter revealed no significant difference between the results from using MyelTracer and those from manual quantification (Fig. 2B, MyelTracer: slope 0.1046, y-intercept $0.7372, n=193$, manual: slope 0.1001 , y-intercept $0.7427, n=193$, slopes $p=0.8311$, intercept $\left.p=0.4695^{\mathrm{d}}\right)$. We did not find a significant difference in $g$-ratio (Fig. 2C, MyelTracer mean \pm SD: $0.7976 \pm 0.04328, n=193$, manual: $\left.0.8018 \pm 0.04244, p=0.3406^{\mathrm{e}}\right)$, but a significant reduction in time spent quantifying $g$-ratio (Fig. $2 D$, MyelTracer mean: 0.4808, manual: mean 1.0, $n=193$ axons, three images, $\left.p=0.0046^{f}\right)$. In the sciatic nerve of one-month-old mice, regression relationships between $g$-ratio and axon diameter were not significantly different between using MyelTracer and manual quantification (Fig. $2 F$, MyelTracer: slope -0.0386 , y-intercept $0.627, n=104$, manual: slope $-0.030, y$-intercept $0.615, n=104$, slopes $p=0.7437$, intercept $p=0.8997^{9}$ ). Comparing the results from using MyelTracer and those from manual quantification in the sciatic nerve, there was no difference in $g$-ratio (Fig. $2 G$, MyelTracer mean \pm SD: $0.5586 \pm 0.07121, n=104$, manual: $\left.0.5576 \pm 0.06833, p=0.9146^{\mathrm{h}}\right)$, while there was a significant reduction in time spent quantifying $g$-ratio (Fig. $2 H$, MyelTracer mean 0.4054 , manual mean 1 , $n=104$ axons, 6 images, $\left.p<0.0001^{i}\right)$. Together, these findings demonstrate that MyelTracer saves $40-60 \%$ user quantification time and offers accuracy levels that are comparable to manual quantification.

\section{Myelin thickness abnormalities can be detected using MyelTracer}

Using MyelTracer for $g$-ratio quantification saves a significant amount of time and produces accurate results across different types of tissues from wild-type mice (Figs. 1, 2). In research settings, however, most investigators seek a tool to detect differences between genotypes or treatment paradigms. To test whether MyelTracer is suitable for detecting differences 
A

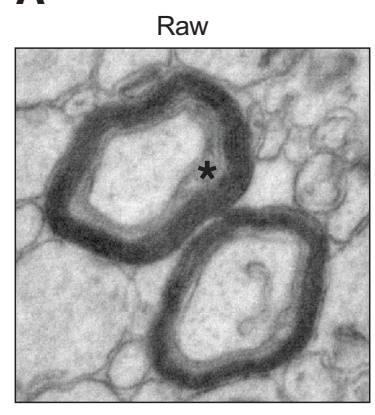

E

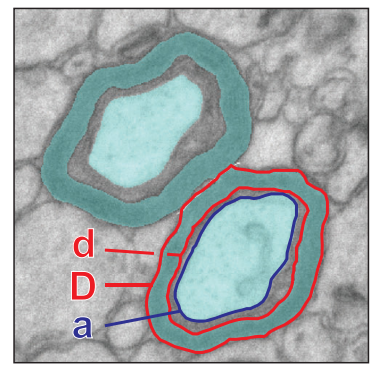

I

1M WT Corpus Callosum

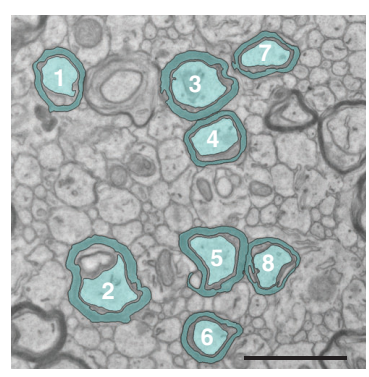

M

Myelin fold

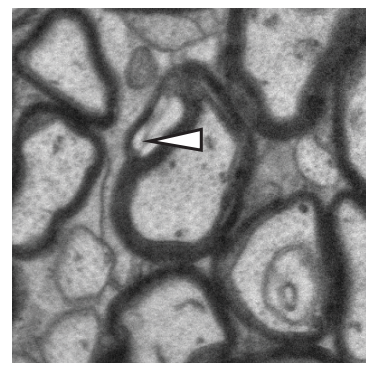

B

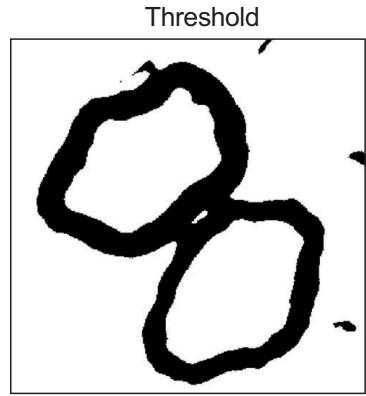

$\mathbf{F}$

g-ratio calculation and plotting

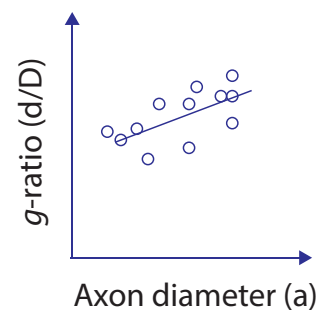

J
C

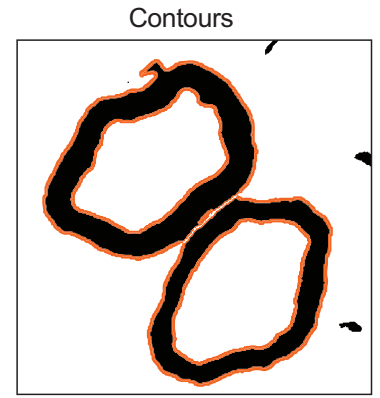

G

GUI

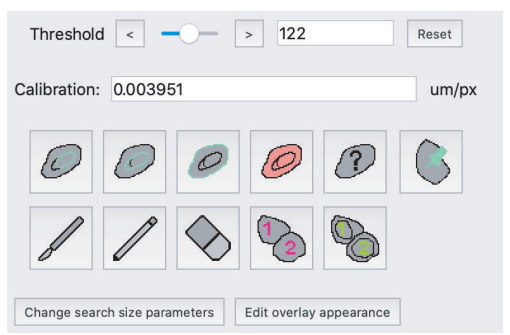

K
D

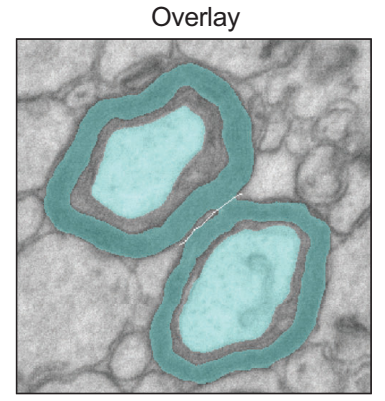

H

Workflow example

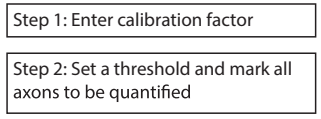

Step 3: Use cut/draw tools to separate/connect myelin sheets

Step 4: Change threshold and mark inner and outer myelin boundaries at once for each axon

Step 5: Save ROIs and export results

$\mathbf{L}$

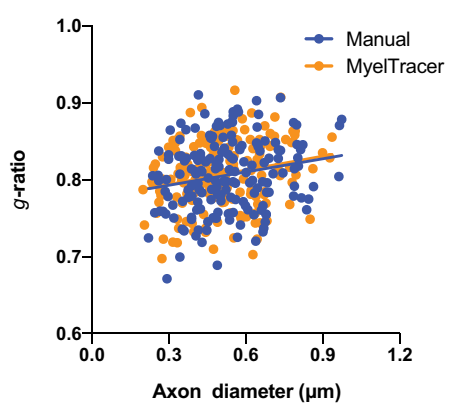

$\mathbf{N}$

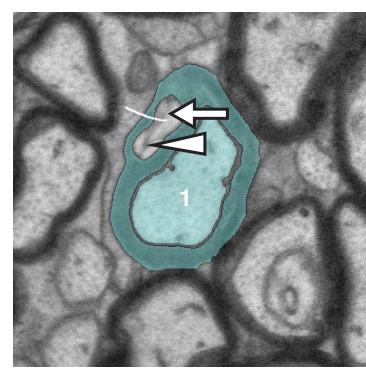

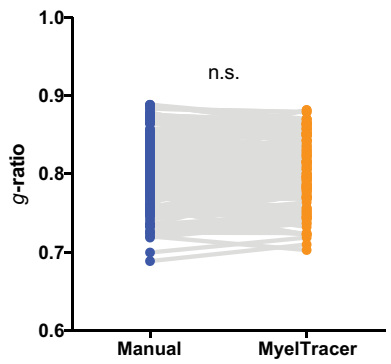

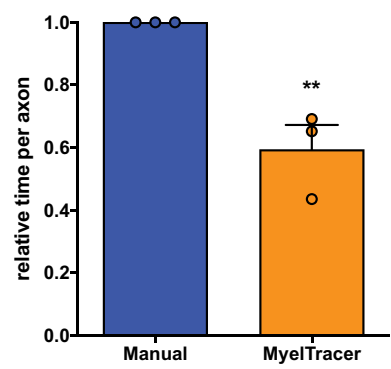

Figure 1. MyelTracer semi-automatically traces axons and myelin and returns results consistent with manual tracing. $\boldsymbol{A}-\boldsymbol{D}$, Electron micrograph of dorsal corpus callosum, software-generated threshold overlays, and postselection overlay (blue) show different steps of the image analysis. * periaxonal space. $\boldsymbol{E}$, Electron micrograph with MyelTracer overlay and outlines for features including axon diameter (a), inner myelin diameter (d), and outer myelin diameter (D), each computed from the respective areas. $\boldsymbol{F}$, Schematic for $\boldsymbol{g}$ ratio scatterplot illustrating how MyelTracer calculates the $g$-ratio. $\mathbf{G}$, Image of the main user interface of MyelTracer. $\boldsymbol{H}$, Schematic showing an example workflow using MyelTracer. I, Representative electron micrograph of corpus callosum from one-month-old wild-type mice. $\boldsymbol{J}$, G-ratio scatter plot from manual and MyelTracer quantifications for the same axons. ANCOVA test $p=0.8996$ (slopes) and $p=0.7044$ (intercepts), $n=174$ axons. $K$, G-ratio from manual and MyelTracer quantification paired for the same axons. Student's $t$ test $p=0.9045, n=174$ axons. $L$, Comparison of time consumption between manual quantification and quantification using MyelTracer. Individual data points represent the average time spent per axon per image; $p=0.0069$; Student's $t$ test. Scale 
continued

bar: $1 \mu \mathrm{m}$. $\boldsymbol{M}-\boldsymbol{P}$, Illustration of MyelTracer's ability to quantify myelin sheets with folds (arrowheads). Folds require the user to draw a white line (arrows) with the cut tool to connect the area inside the fold with the outside area for semi-automated detection. Scale bar: $1 \mu \mathrm{m}$. ns, no significance. ${ }^{* *} p<0.01$.

between two experimental groups, its performance was tested using electron micrographs from control (Gtf2ifl/fl, Nex-Cre-/-) and Gtf2ifflfil Nex-Cre ${ }^{+/-}$mice, a model for Williams syndrome with a known hypomyelination phenotype (Barak et al., 2019). With their genotypes blinded, electron micrographs of corpus callosum from both groups at one month of age were analyzed using MyelTracer (Fig. $3 A, B$ ). Regression relationships between $g$-ratio and axon diameter were significantly different between control and Gtf2if/ffl; Nex$\mathrm{Cre}^{+/-}$mice (Fig. 3C, control: slope 0.1649, y-intercept $0.6447, n=332$ axons, $n=3$ mice, Gtf2if/fif; Nex-Cre ${ }^{+/-}$: slope 0.1487 , y-intercept $0.7132, n=787, n=3$ mice, slopes $p=0.1484$, intercept $\left.p<0.001^{1}\right)$. The $g$-ratio measurements were higher in $\mathrm{Gtf}^{\mathrm{f}} \mathrm{f}^{\mathrm{f} / \mathrm{fl}} ; \mathrm{Nex}-\mathrm{Cre}^{+/-}$mice than those in control mice, indicating a reduction in myelin sheath thickness, a result consistent with the original report (Barak et al., 2019). Together, these data demonstrate that MyelTracer is suitable for detecting differences in myelin $g$-ratios across different genotypes.

\section{MyelTracer can be used to quantify $g$-ratios in remyelination}

MyelTracer readily recognizes well-developed myelin sheaths in micrographs of both central and peripheral nerves and axon tracts. In contrast to the relatively thick and compact myelin in these tissues, myelin in remyelinating tissues after injury is characterized by a thinner morphology (Franklin and ffrench-Constant, 2008; Duncan et al., 2017). To test MyelTracer's ability to quantify $g$-ratios in tissues undergoing remyelination, we analyzed micrographs from an optic nerve crush model $28 \mathrm{~d}$ after the injury (Fig. 3D). Comparing the results from MyelTracer and those from manual quantification in this model, there was no difference in $g$-ratio (Fig. $3 E$,
A $2 M$ WT Optic Nerve
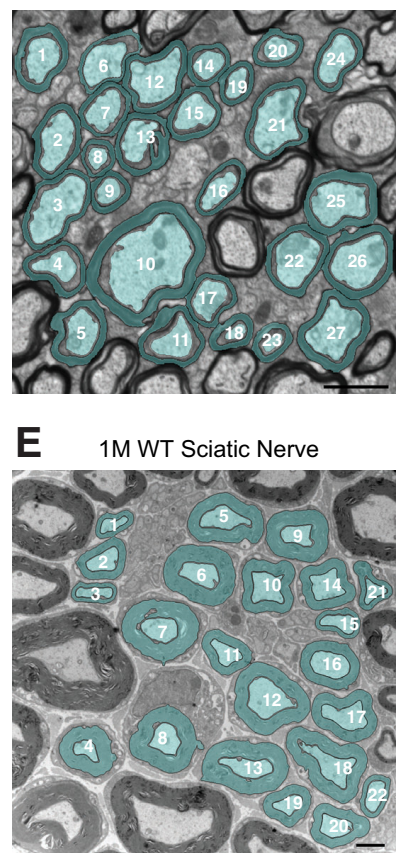

B

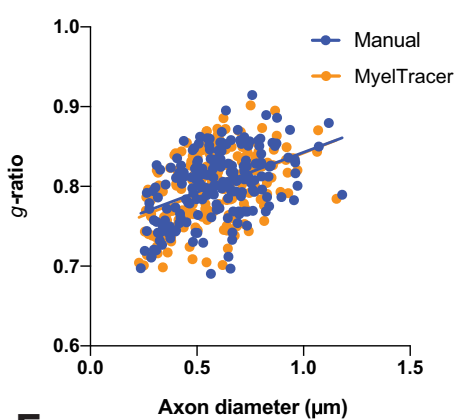

$\mathbf{F}$

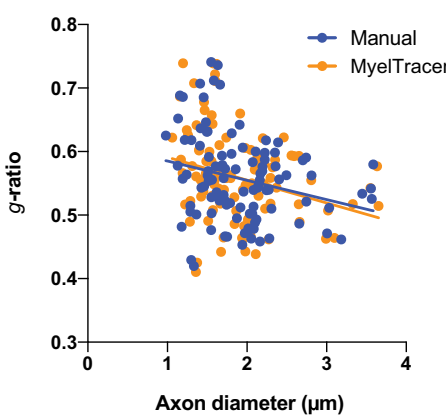

C

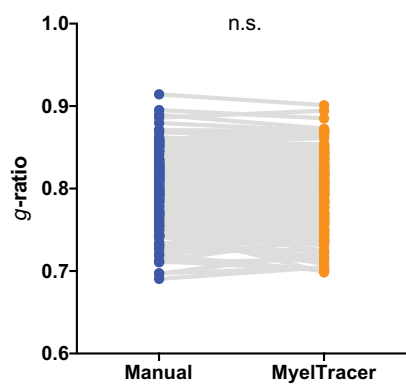

G

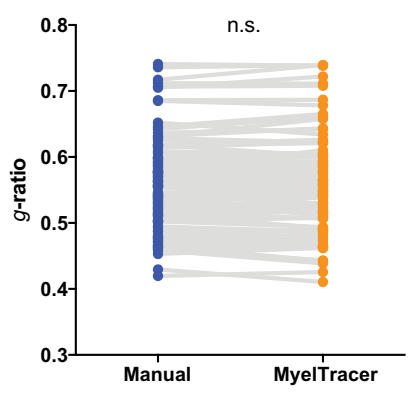

D

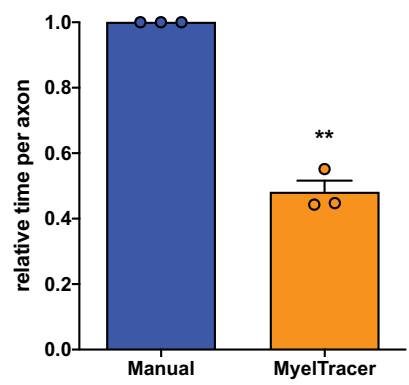

H

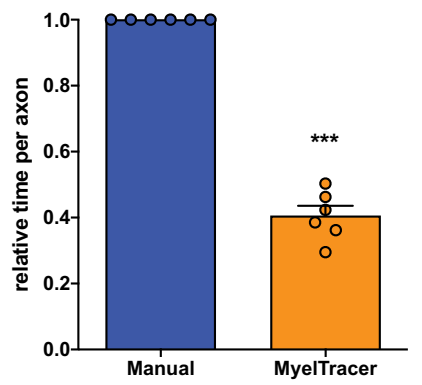

Figure 2. MyelTracer accurately returns $g$-ratios for myelinated tissues of varying axonal density. $\boldsymbol{A}$, Representative electron micrograph of the optic nerve at two months of age $(2 \mathrm{M})$ with MyelTracer-generated overlays (blue). Scale bar: $1 \mu \mathrm{m}$. $\boldsymbol{B}, g$-Ratio scatter plot from manual and MyelTracer quantifications for the same axons. ANCOVA test $p=0.8311$ (slopes) and $p=0.4695$ (intercepts), $n=193$ axons. $\boldsymbol{C}, g$-Ratios from manual and MyelTracer quantifications paired for the same axons. $p=0.3406, n=193$ axons. $\boldsymbol{D}$, Comparison of time consumption between manual quantification and quantification using MyelTracer. Individual data points represent the average time spent per axon per image; $p=0.0046$. $\boldsymbol{E}$, Representative electron micrograph of the sciatic nerve at one month of age with MyelTracer-generated overlays (blue). Scale bar: $2 \mu \mathrm{m}$. $\boldsymbol{F}, g$-Ratio scatter plot from manual and MyelTracer quantifications for the same axons. ANCOVA test $p=0.7437$ (slopes) and $p=0.8997$ (intercepts), $n=104$ axons. G, $g$-Ratios from manual and MyelTracer quantifications paired for the same axons. $p=0.9146, n=104$ axons. $\boldsymbol{H}$, Comparison of time consumption between manual quantification and quantification using MyelTracer. Individual data points represent the average time spent per axon per image; $p<0.0001$; Student's $t$ test. ns, no significance. ${ }^{\star *} p<0.01,{ }^{* *} p<0.001$. 
A

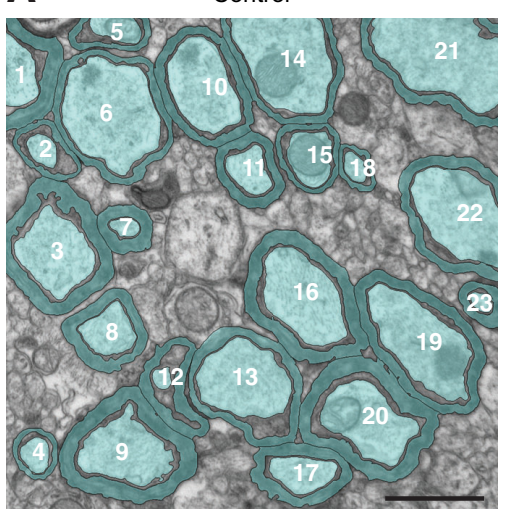

Remyelination after optic nerve crush

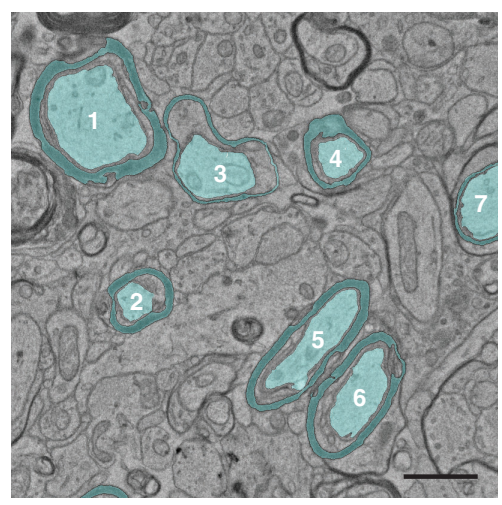

G Native optic nerve (no crush)

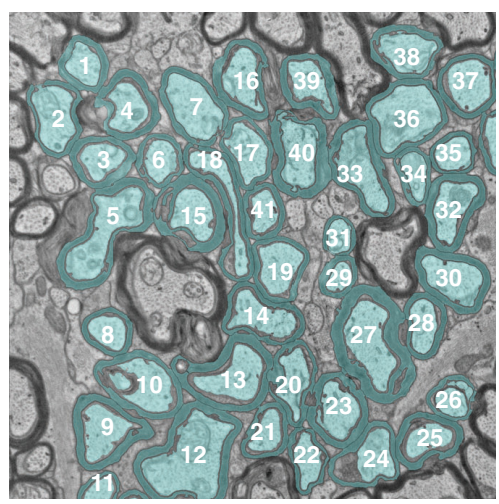

B

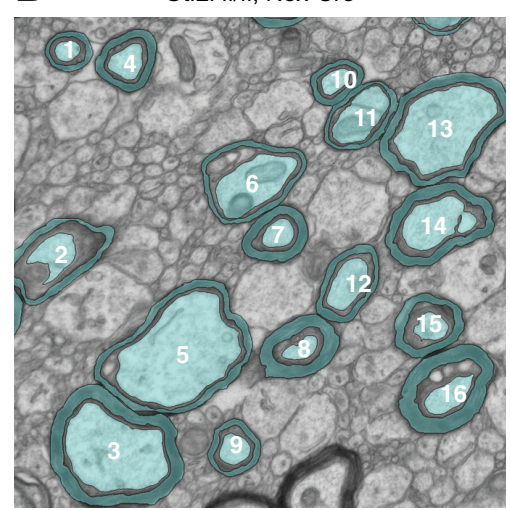

E

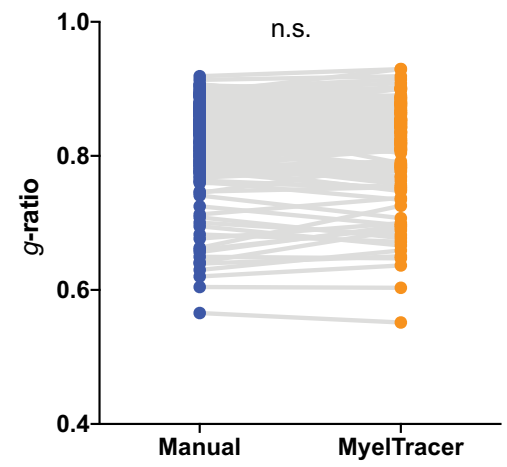

H

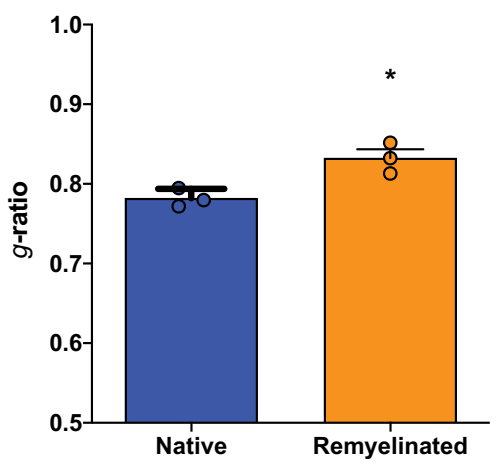

C

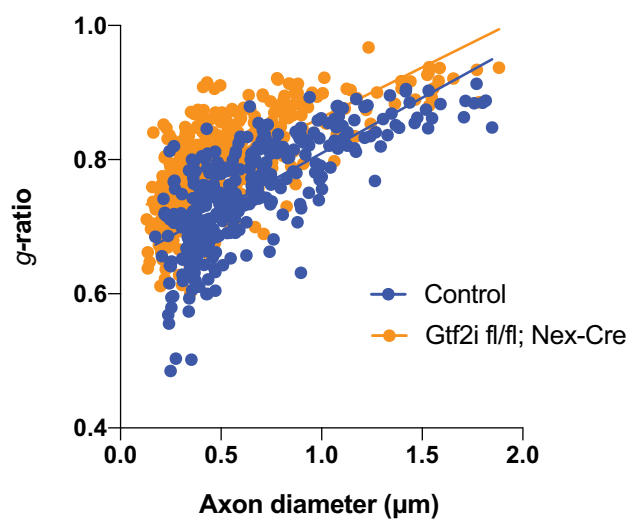

$\mathbf{F}$
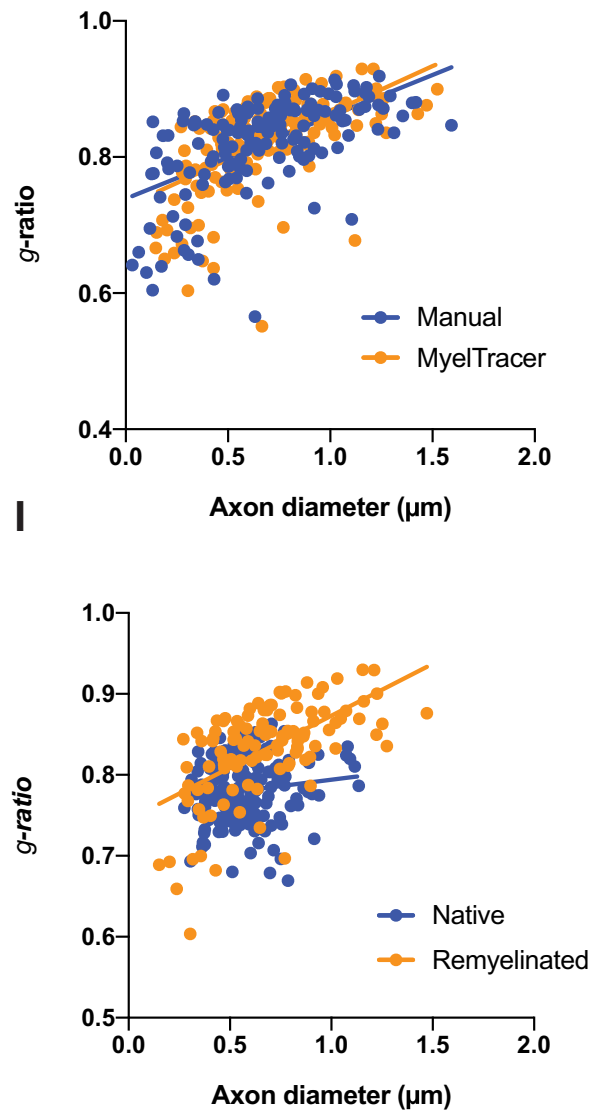

Figure 3. MyelTracer can be used to detect a myelin thickness abnormality in a Williams syndrome mouse model and to measure $g$-ratios in remyelination. $\boldsymbol{A}, \boldsymbol{B}$, Representative electron micrographs of the corpus callosum in control and Gtf2i fl/fl; Nex-Cre mice at one month of age with MyelTracer-generated overlays (blue). Scale bar: $1 \mu \mathrm{m}$. C, G-ratio scatter plot from using MyelTracer for control and Gtf2i fl/fl; Nex-Cre mice; $p<0.0001$; ANCOVA test; $n=3$ mice, 332 axons for control and $n=3$ mice, 455 axons for Gtf2i $\mathrm{fl} / \mathrm{fl}$; Nex-Cre mice. $\boldsymbol{D}$, Representative electron micrograph of the optic nerve $28 \mathrm{~d}$ after optic nerve crush with MyelTracer-generated overlays (blue). Scale bar: $1 \mu \mathrm{m}$. $\boldsymbol{E}$, G-ratio from manual and MyelTracer quantifications paired for the same axons. $p=0.9699$, $n=155$ axons. $\boldsymbol{F}$, G-ratio scatter plot from manual and MyelTracer quantifications for the same axons. ANCOVA test $p=0.1511$ (slopes) and $p=0.8686$ (intercepts), $n=155$ axons. G, Representative electron micrograph of the native control optic nerve with MyelTracer-generated overlays (blue). $\boldsymbol{H}$, G-ratio group comparison of axons from native control and remyelinated axons from mice following optic nerve crush; $p<0.0183$; $t$ test; $n=3$ mice per group. I, G-ratio scatter plot of axons from native control and remyelinated axons from mice following optic nerve crush. ANCOVA test $p<0.0001$ (slopes), native control $n=183$ axons, remyelinated $n=116$ axons. ns, no significance. ${ }^{*} p<0.05$. 
MyelTracer mean \pm SD: $0.8212 \pm 0.06843, n=155$, manual: $\left.0.8210 \pm 0.06944, p=0.9699^{k}\right)$. Further, regression relationships between $g$-ratio and axon diameter were not significantly different between MyelTracer and manual quantification (Fig. 3F, MyelTracer: slope 0.1484, y-intercept $0.7150, n=155$, manual: slope 0.1282 , y-intercept $0.7273, n=155$, slopes $p=0.1511$, intercept $p=0.8686^{\prime}$ ). To further test whether MyelTracer is suitable to detect the thinned myelin sheets in remyelination compared with native myelin sheets, its performance was tested using region-matched electron micrographs from remyelinated axons and axons from matched native control animals (Fig. 3G). Comparison of the average g-ratio per animal revealed significantly increased $g$-ratios in the remyelinated group compared with the native control group (Fig. $3 H$, native control mean \pm SD: $0.7821 \pm 0.01162, n=3$ mice, remyelinated: $0.8324 \pm 0.01942, n=3$ mice, $p=$ $\left.0.0183^{\mathrm{m}}\right)$. Regression relationships between $g$-ratio and axon diameter were significantly different between the optic nerve axons in native control and remyelinated mice (Fig. 3/, native control: slope 0.03137, y-intercept 0.7627, $n=183$ axons, remyelinated: slope 0.1281 , y-intercept $0.7449, n=116, n=3$ mice, slopes $\left.p<0.0001^{1}\right)$. Together, these results indicate that MyelTracer is suitable for $g$-ratio measurements in remyelination.

\section{Discussion}

In this study, we show that MyelTracer can address the need for an easy-to-use, stand-alone software for streamlining $g$-ratio quantification. OpenCV, PyQt5, and fbs were used in Python to develop and package MyelTracer, an easy to install software with an intuitive GUI. MyelTracer utilizes contrast in electron micrographs to generate contours around axons and myelin sheaths. The user can select contours outlining myelin features of interest (axons, inner myelin, and outer myelin), which then allow MyelTracer to group corresponding features, measure their areas and calculate $g$-ratios. As an all-in-one platform for myelin analyses, MyelTracer can also be used to calculate the percentage of myelinated axons. Using MyelTracer, user quantification time is reduced by $40-$ $60 \%$ depending on the region, and its accuracy is comparable to manual tracing. Using MyelTracer, a known hypomyelination phenotype of the Williams syndrome mouse model can be detected, confirming its suitability for studies assessing phenotypic changes in myelin sheaths. MyelTracer is freely available at https://github.com/ HarrisonAllen/MyelTracer.

Myelin ultrastructure is highly heterogeneous with a wide range of axon calibers and myelin sheath morphologies, presenting a challenge for automated analysis. MyelTracer uses OpenCV to automatically contour crosssections of axons and myelin sheaths in electron micrographs (Fig. $1 A-C)$. The easy-to-use GUI allows users to intuitively interact with and fine-tune the results generated by the underlying computer vision algorithms (Fig. 1D,G). MyelTracer decreases analysis time by dramatically accelerating axon and myelin sheath contouring compared with manual tracing. This automated feature differentiates MyelTracer from existing non-automated tools, such as
GRatio for ImageJ, which is an ImageJ plugin that facilitates grouping of traced structures and data export (Goebbels et al., 2010).

In addition to MyelTracer, several semi-automated and automated tools are available that exhibit different strengths and weaknesses. AxonSeg and AxonDeepSeg are MATLAB-based segmentation algorithms that are fully automated, enabling high-throughput measurements (Zaimi et al., 2016, 2018). These tools are useful for processing large datasets but require image preprocessing, adjustment of parameters in MATLAB, or additional training of the neural network using manually labeled images from user-specific datasets, which can be hurdles for implementation. Janjic et al. (2019) developed a high-fidelity tool using deep neural networks, which suits researchers with a computer science background seeking an advanced $g$-ratio quantification tool. While there are other useful tools available, MyelTracer's intuitive GUI makes it a powerful tool allowing researchers to quantify myelin without computer science knowledge.

G-ratio quantification using MyelTracer was demonstrated to be accurate across different tissues, such as corpus callosum, optic nerve, and sciatic nerve (Figs. 1, 2). For these types of samples, there was a $40-60 \%$ reduction in time spent on quantification (Figs. $1 L, 2 D, H$ ). The variation in the amount of time-reduction depended on the type of tissue. The smallest time-reduction was for corpus callosum of one-month-old mice. This is likely because of the heterogeneity in axonal caliber, axonal density, and the contrast level among the axon, periaxonal space, inner tongue, and the myelin sheath (Fig. $1 A, I)$. In contrast, the greatest time-reduction was observed for sciatic nerve, which has clearly defined myelinated axons with homogeneous morphology, minimal periaxonal space and minimal myelin contiguity between adjacent axons (Fig. 2E,H). Comparing MyelTracer workflows for corpus callosum versus sciatic nerve, a major difference was that the latter required less manual input to separate contiguous myelin sheaths (step 3; Fig. 1H). Similar to manual tracing and other computational toolboxes, MyelTracer also relies on the quality of input images and therefore shows less effective performance for images with poor contrast levels, artifacts from inadequate perfusion or tissue handling.

The analysis of electron micrographs from Williams syndrome mice shows that MyelTracer readily detects abnormalities in myelination (Fig. $3 A-C$ ), demonstrating the suitability of MyelTracer for detecting differences in myelin g-ratio across different genotypes or treatment groups. In such studies, MyelTracer will decrease user quantification time, and streamline data generation for publication purposes.

Extending the application for MyelTracer, analysis of remyelinating axons $28 \mathrm{~d}$ after optic nerve crush shows that the software is suitable for $g$-ratio measurements in studies of remyelination (Fig. $3 D-l$ ). Myelin sheaths in remyelinating tissues are generally found to be thinner (Franklin and ffrench-Constant, 2008; Duncan et al., 2017), and they can appear almost discontinuous in a given sectioning plane. This presents a challenge 
to automation and may require user input using MyelTracer's Cut and Draw tools as extensively outlined in the Users' Manual (Extended Data 2). Notwithstanding these challenges, MyelTracer is suitable for the semi-automated tracing of micrographs in studies of remyelination provided that the myelin sheaths and axons display good contrast.

As a user-friendly all-in-one software suite, MyelTracer will also allow researchers to quantify the percentage of myelinated axons, which together with $g$-ratio, are the most commonly reported metrics in myelin studies. Beyond existing tools and MyelTracer, advances in computer vision and artificial intelligence may further advance data analysis methods and produce more powerful toolboxes to streamline quantitative analysis of myelin ultrastructure.

\section{References}

Barak B, Zhang Z, Liu Y, Nir A, Trangle SS, Ennis M, Levandowski KM, Wang D, Quast K, Boulting GL, Li Y, Bayarsaihan D, He Z, Feng G (2019) Neuronal deletion of Gtf2i, associated with Williams syndrome, causes behavioral and myelin alterations rescuable by a remyelinating drug. Nat Neurosci 22:700-708.

Bégin S, Dupont-Therrien O, Bélanger E, Daradich A, Laffray S, De Koninck Y, Côté DC (2014) Automated method for the segmentation and morphometry of nerve fibers in large-scale CARS images of spinal cord tissue. Biomed Opt Express 5:4145-4161.

Duncan ID, Radcliff AB (2016) Inherited and acquired disorders of myelin: the underlying myelin pathology. Exp Neurol 283:452-475.

Duncan ID, Marik RL, Broman AT, Heidari M (2017) Thin myelin sheaths as the hallmark of remyelination persist over time and preserve axon function. Proc Natl Acad Sci USA 114:E9685-E9691.

Franklin RJM, ffrench-Constant C (2008) Remyelination in the CNS: from biology to therapy. Nat Rev Neurosci 9:839-855.

Gibson EM, Purger D, Mount CW, Goldstein AK, Lin GL, Wood LS, Inema I, Miller SE, Bieri G, Zuchero JB, Barres BA, Woo PJ, Vogel $\mathrm{H}$, Monje M (2014) Neuronal activity promotes oligodendrogenesis and adaptive myelination in the mammalian brain. Science 344:1252304.

Goebbels S, Oltrogge JH, Kemper R, Heilmann I, Bormuth I, Wolfer S, Wichert SP, Möbius W, Liu X, Lappe-Siefke C, Rossner MJ, Groszer M, Suter U, Frahm J, Boretius S, Nave K-A (2010) Elevated phosphatidylinositol 3, 4, 5-trisphosphate in glia triggers cell-autonomous membrane wrapping and myelination. $J$ Neurosci 30:8953-8964.

Hughes EG, Appel B (2016) The cell biology of CNS myelination. Curr Opin Neurobiol 39:93-100.

Hughes EG, Orthmann-Murphy JL, Langseth AJ, Bergles DE (2018) Myelin remodeling through experience-dependent oligodendrogenesis in the adult somatosensory cortex. Nat Neurosci 21:696706.

Janjic P, Petrovski K, Dolgoski B, Smiley J, Zdravkovski P, Pavlovski G, Jakjovski Z, Davceva N, Poposka V, Stankov A, Rosoklija G, Petrushevska G, Kocarev L, Dwork AJ (2019) Measurement-oriented deep-learning workflow for improved segmentation of myelin and axons in high-resolution images of human cerebral white matter. J Neurosci Methods 326:108373.

Krajewski KM, Lewis RA, Fuerst DR, Turansky C, Hinderer SR, Garbern J, Kamholz J, Shy ME (2000) Neurological dysfunction and axonal degeneration in Charcot-Marie-Tooth disease type 1A. Brain 123:1516-1527.

Lin W, Popko B (2009) Endoplasmic reticulum stress in disorders of myelinating cells. Nat Neurosci 12:379-385.

Mayoral SR, Chan JR (2016) The environment rules: spatiotemporal regulation of oligodendrocyte differentiation. Curr Opin Neurobiol 39:47-52.

McKenzie IA, Ohayon D, Li H, De Faria JP, Emery B, Tohyama K, Richardson WD (2014) Motor skill learning requires active central myelination. Science 346:318-322.

More HL, Chen J, Gibson E, Donelan JM, Beg MF (2011) A semi-automated method for identifying and measuring myelinated nerve fibers in scanning electron microscope images. J Neurosci Methods 201:149-158.

Nave KA (2010) Myelination and support of axonal integrity by glia. Nature 468:244-252.

Phan BN, Bohlen JF, Davis BA, Ye Z, Chen HY, Mayfield B, Sripathy SR, Cerceo Page S, Campbell MN, Smith HL, Gallop D, Kim H, Thaxton CL, Simon JM, Burke EE, Shin JH, Kennedy AJ, Sweatt JD, Philpot BD, Jaffe AE, et al. (2020) A myelin-related transcriptomic profile is shared by Pitt-Hopkins syndrome models and human autism spectrum disorder. Nat Neurosci 23:375-385.

Rushton WAH (1951) A theory of the effects of fibre size in medullated nerve. J Physiol 115:101-122.

Salzer JL (2015) Schwann cell myelination. Cold Spring Harb Perspect Biol 7:a020529.

Sander S, Ouvrier R, McLeod J, Nicholson G, Pollard J (2000) Clinical syndromes associated with tomacula or myelin swellings in sural nerve biopsies. J Neurol Neurosurg Psychiatry 68:483488.

Sherman DL, Brophy PJ (2005) Mechanisms of axon ensheathment and myelin growth. Nat Rev Neurosci 6:683-690.

Simons M, Trotter J (2007) Wrapping it up: the cell biology of myelination. Curr Opin Neurobiol 17:533-540.

Stadelmann C, Timmler S, Barrantes-Freer A, Simons M (2019) Myelin in the central nervous system: structure, function, and pathology. Physiol Rev 99:1381-1431.

Sun LO, Mulinyawe SB, Collins HY, Ibrahim A, Li Q, Simon DJ, Tessier-Lavigne M, Barres BA (2018) Spatiotemporal control of CNS myelination by oligodendrocyte programmed cell death through the TFEB-PUMA axis. Cell 175:1811-1826.

Wang J, He X, Meng H, Li Y, Dmitriev P, Tian F, Page JC, Lu QR, He $Z$ (2020) Robust myelination of regenerated axons induced by combined manipulations of GPR17 and microglia. Neuron 108:876-886.

Zaimi A, Duval T, Gasecka A, Côté D, Stikov N, Cohen-Adad J (2016) AxonSeg: open source software for axon and myelin segmentation and morphometric analysis. Front Neuroinform 10:37.

Zaimi A, Wabartha M, Herman V, Antonsanti PL, Perone CS, CohenAdad J (2018) AxonDeepSeg: automatic axon and myelin segmentation from microscopy data using convolutional neural networks. Sci Rep 8:3816.

Zhao C, Dong C, Frah M, Deng Y, Marie C, Zhang F, Xu L, Ma Z, Dong X, Lin Y, Koenig S, Nait-Oumesmar B, Martin DM, Wu LN, Xin M, Zhou W, Parras C, Lu QR (2018) Dual requirement of CHD8 for chromatin landscape establishment and histone methyltransferase recruitment to promote CNS myelination and repair. Dev Cell 45:753-768. 